\title{
Alterações psicológicas durante a pandemia por COVID-19 e sua relação com bruxismo e DTM
}

Psychological changes during the COVID-19 pandemic and its relationship with bruxism and TMD

Cambios psicológicos durante la pandemia de COVID-19 y su relación con bruxismo y TMD

Jessilene Ribeiro Rocha

ORCID: https://orcid.org/0000-0002-5681-5722 Universidade Federal do Maranhão, Brasil

E-mail: jessilene.rr@gmail.com

Myllena Jorge Neves

ORCID: https://orcid.org/0000-0002-2186-5340 Universidade Federal do Maranhão, Brasil

E-mail: myllenajorgen@gmail.com

Maryana Romana Rocha Pinheiro

ORCID: https://orcid.org/0000-0003-2628-7030

Universidade Federal do Maranhão, Brasil

E-mail: maryanaromana5@gmail.com

Maria Áurea Lira Feitosa

ORCID: https://orcid.org/0000-0002-9177-2369

Universidade Federal do Maranhão, Brasil

E-mail: aurea.maria@ufma.br

Rosana Costa Casanovas

ORCID: https://orcid.org/0000-0002-6871-3491 Universidade Federal do Maranhão, Brasil

E-mail: rosana.casanovas@ufma.br

Darlon Martins Lima

ORCID: https://orcid.org/0000-0001-9113-1206 Universidade Federal do Maranhão, Brasil

E-mail: darlon.martins@ufma.br

\section{Resumo}

A pandemia do coronavírus ocasionou diversas mudanças na vida da população, causando consequências psicológicas que podem influenciar na saúde bucal, principalmente doenças orais que têm como etiologia fatores psicoemocionais. Objetivo: realizar uma revisão de literatura acerca da influência da pandemia por COVID-19 nos aspectos psicológicos e sua relação com o bruxismo e a Disfunção Temporomandibular (DTM). Metodologia: trata-se de uma revisão bibliográfica de caráter qualitativo em que se realizou a coleta de dados nas plataformas LILACS, BVS, SciELO e PubMed usando os descritores COVID-19, Oral Health, Psychology, Anxiety, Depression, Bruxism, Temporomandibular Disorders em língua inglesa e portuguesa, sendo incluídos artigos que abordassem a associação entre aspectos psicossociais relacionados à pandemia por COVID-19 e sua ligação com o bruxismo e a DTM, além de artigos que correlacionavam a DTM e os aspectos psicológicos ou que tratavam sobre a pandemia por COVID-19 e sua ligação com aspectos psicológicos. Resultados: foram selecionados 19 artigos e estes evidenciaram uma relação entre estresse, ansiedade e depressão com bruxismo e DTM, sendo as mulheres as mais acometidas. Conclusão: a pandemia por COVID-19 elevou os níveis de estresse, ansiedade e depressão, assim como a incidência e a severidade dos sintomas dolorosos de bruxismo e DTM.

Palavras-chave: Coronavírus; DTM; Saúde mental; Isolamento social.

\section{Abstract}

The coronavirus pandemic caused several changes in the population's life, causing psychological consequences that can influence oral health, especially oral diseases that have psycho-emotional factors as their etiology. Objective: perform a literature review about the influence of the COVID-19 pandemic on psychological aspects and its relationship with bruxism and Temporomandibular Disorder (TMD). Methodology: this is a qualitative bibliographic review in which data collection was carried out on the LILACS, BVS, SciELO and PubMed platforms using the descriptors: COVID-19, Oral Health, Psychology, Anxiety, Depression, Bruxism, Temporomandibular Disorders, in English and Portuguese, including articles that addressed the association between psychosocial aspects related to the pandemic by COVID-19 and its connection with bruxism and TMD, in addition to articles that correlated TMD and psychological aspects or that dealt with the pandemic by COVID-19 
and its connection with psychological aspects. Results: 19 articles were selected and these showed a relationship between stress, anxiety and depression with bruxism and TMD, with women being the most affected. Conclusion: the COVID-19 pandemic increased levels of stress, anxiety and depression, as well as the incidence and severity of painful symptoms of bruxism and TMD.

Keywords: Coronavirus; TMD; Mental health; Social isolation.

\section{Resumen}

La pandemia de coronavirus provocó varios cambios en la vida de la población, provocando consecuencias psicológicas que pueden influir en la salud bucal, especialmente enfermedades bucales que tienen como etiología factores psicoemocionales. Objetivo: realizar una revisión de la literatura sobre la influencia de la pandemia COVID-19 en los aspectos psicológicos y su relación con el bruxismo y el Trastorno Temporomandibular (TTM). Metodología: se trata de una revisión bibliográfica cualitativa en la que se recogieron datos de las plataformas LILACS, BVS, SCIELO y PUBMED utilizando los descriptores: COVID-19, Salud Bucal, Psicología, Ansiedad, Depresión, Bruxismo, en inglés y portugués, incluyendo artículos que abordaron la asociación entre aspectos psicosociales relacionados con la pandemia por COVID-19 y su conexión con bruxismo y TMD, además de artículos que correlacionaron TMD y aspectos psicológicos o que abordaron la pandemia por COVID-19 y su conexión con los aspectos psicológicos. Resultados: Se seleccionaron 19 artículos y estos mostraron una relación entre el estrés, la ansiedad y la depresión con el bruxismo y el TMD, siendo las mujeres las más afectadas. Conclusión: la pandemia de COVID-19 incrementó los niveles de estrés, ansiedad y depresión, así como la incidencia y severidad de los síntomas dolorosos del bruxismo y TMD.

Palabras clave: Coronavirus; TMD; Salud mental; Aislamiento social.

\section{Introdução}

A COVID-19 (Coronavirus Disease 2019) é uma doença contagiosa causada pelo vírus Sars-Cov-2, sendo identificada pela primeira vez em dezembro de 2019 na cidade de Wuhan, na China (OPAS, 2020). Devido à propagação do vírus em nível mundial, em março de 2020 a Organização Mundial de Saúde (OMS) declarou a doença como uma pandemia (Ather, 2020). A transmissão do vírus se dá por meio de gotículas expelidas através de espirro, tosse ou contato com superfícies contaminadas (Izzetti, 2020). Os sintomas mais frequentes da doença são cansaço, tosse seca e febre, mas outras manifestações como dor de cabeça, dor de garganta, diarreia, perda de paladar e/ou olfato e até erupção cutânea na pele podem estar presentes (OPAS, 2020).

Em razão da alta taxa de transmissão do vírus, foram adotadas diversas medidas, visando diminuir a contaminação pela doença. Além da recomendação de constante higiene pessoal, como uso de álcool em gel, lavagem das mãos e uso de máscara, outras maneiras de controle foram tomadas por entidades governamentais, objetivando a contenção da pandemia (Aquino et al., 2020).

Dentre elas, o distanciamento social busca limitar o convívio social, por meio do fechamento de espaços coletivos, evitando aglomerações. O isolamento social é uma estratégia em que se recomenda o afastamento de pessoas infectadas das não contaminadas. Em casos mais rigorosos, onde apenas os serviços essenciais são mantidos, o distanciamento social é chamado de lockdown (bloqueio). A quarentena, por sua vez, isola as pessoas que possivelmente foram expostas ao vírus, mesmo que estas não apresentem sintomas. A ausência de sintomatologia pode ocorrer tanto por não estarem infectados, quanto por estarem no período de incubação do vírus ou até mesmo por serem assintomáticas (Pereira et al., 2020).

Neste cenário de mudança da rotina, afastamento dos amigos e/ou familiares e aumento do medo gerado pela doença há uma tendência a consequências negativas na saúde mental da população (Pereira et al., 2020; Tassara et al., 2021). A perda de conexão entre as pessoas acaba por aumentar os anseios e sentimentos de raiva e solidão (Almeida-Leite; StuginskiBarbosa; Conti, 2020).

O estresse causado pelo isolamento social, o grande número de informações, muitas vezes controversas e a preocupação gerada pelo receio da doença ou dificuldades de subsistência podem agravar a ansiedade e depressão nas pessoas (Limcaoco et al., 2020), tornando-as mais propensas a problemas bucais associados ao estresse (Almeida, Guimarães, Almeida, 2018). 
Considerando que a sensação de medo e incerteza podem gerar manifestações psicológicas desfavoráveis, e que os fatores psicossociais podem ter relação direta com agravos relacionados à saúde bucal é que esse trabalho tem como objetivo realizar uma revisão de literatura acerca da relação entre problemas psicológicos gerados pela pandemia por COVID-19 e sintomatologias relacionadas ao bruxismo e Disfunção Temporomandibular (DTM).

\section{Metodologia}

Este estudo constitui uma revisão integrativa de caráter qualitativo sobre os agravos em saúde bucal decorrentes de alterações psicológicas durante a pandemia por COVID-19. Buscou-se avaliar como a pandemia por COVID-19 influenciou nos aspectos psicossociais e qual sua relação com o bruxismo e a DTM.

Realizou-se a coleta de dados no mês de abril de 2021, utilizando para a pesquisa as bases de dados Literatura LatinoAmericana e do Caribe em Ciências da Saúde (LILACS), Biblioteca Virtual em Saúde (BVS), Scientific Eletronic Library Online (SciELO) e National Library of Medicine (PubMed). Foram selecionados artigos publicados nos anos de 2017 a 2021, não havendo recorte territorial, empregando os descritores COVID-19, Oral Health, Psychology, Anxiety, Depression, Bruxism, Temporomandibular Disorders em língua inglesa e portuguesa. A busca foi realizada através de título, resumo e palavras-chave.

Os critérios de inclusão foram: artigos que abordassem a associação entre aspectos psicossociais relacionados à pandemia por COVID-19 e sua ligação com o bruxismo e a DTM. Além disso, optou-se por incluir artigos que correlacionavam a DTM e os aspectos psicológicos ou a pandemia por COVID-19 e os aspectos psicológicos.

Excluíram-se os artigos que não tratavam da relação entre a pandemia por COVID-19 e aspectos psicológicos ou não correlacionaram DTM e aspectos psicológicos e, ainda, que não demonstraram a relação entre pandemia e DTM.

Foram identificados 35 artigos e, após leitura do título e resumo, foram excluídos 12 por não atenderem os critérios de inclusão ou por não estarem disponíveis na íntegra. Assim, foram selecionados 23 artigos que foram lidos para confirmar sua elegibilidade. Desses, foram excluídos 4 por não contemplarem as associações estabelecidas, restando 19 trabalhos incluídos nesta revisão.

Figura 1 - Fluxograma de seleção de artigos.

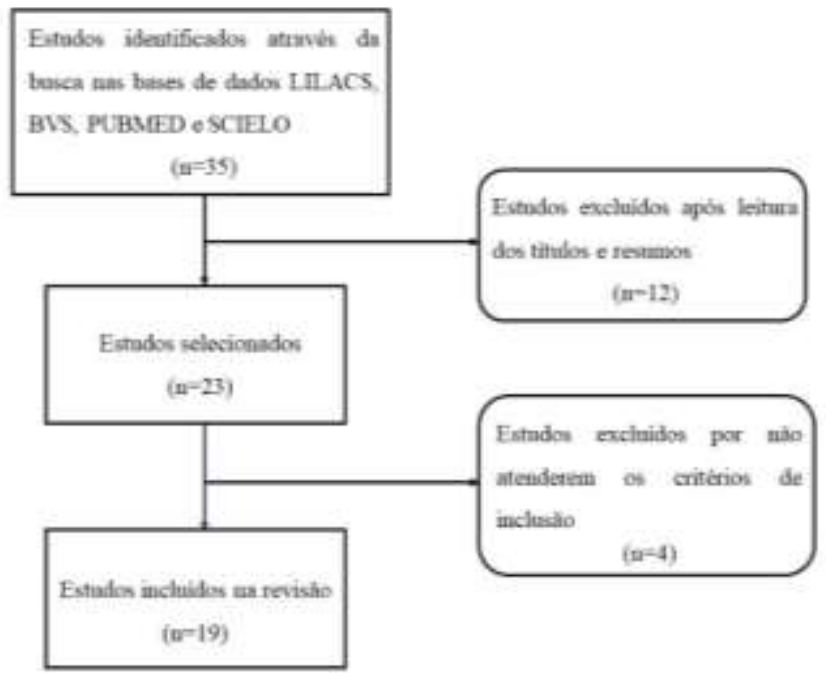

Fonte: Autores. 


\section{Resultados}

Tabela 1 - Associação entre aspectos psicossociais e a pandemia por COVID-19.

\begin{tabular}{|c|c|c|}
\hline Autor, ano & Método e objetivo & Resultados \\
\hline $\begin{array}{l}\text { Di Blasi et al., } \\
2021\end{array}$ & $\begin{array}{l}\text { Pesquisa quantitativa. Investigar os } \\
\text { caminhos que estão por trás de alguns } \\
\text { componentes do sofrimento psicológico } \\
\text { e suas mudanças ao longo do tempo } \\
\text { (durante e após lockdown relacionado à } \\
\text { COVID-19). }\end{array}$ & $\begin{array}{l}\text { Os sintomas depressivos e suas associações com outras dimensões da } \\
\text { angústia individual são um fator chave na compreensão da influência do } \\
\text { surto na saúde mental. As intervenções focadas na melhoria do suporte } \\
\text { social e emocional são estratégias eficazes para minimizar o impacto da } \\
\text { depressão relacionada com a COVID-19. }\end{array}$ \\
\hline $\begin{array}{l}\text { De Boni et al., } \\
2020\end{array}$ & $\begin{array}{l}\text { Pesquisa quantitativa. Avaliar a } \\
\text { prevalência e os preditores de } \\
\text { depressão, ansiedade e e sua } \\
\text { comorbidade entre trabalhadores } \\
\text { essenciais no Brasil e na Espanha } \\
\text { durante a pandemia por COVID-19. }\end{array}$ & $\begin{array}{l}\text { Dentre a população estudada, os indivíduos mais propensos a terem } \\
\text { sintomas de ansiedade ou depressão foram as mulheres jovens } \\
\text { trabalhadoras da linha de frente de combate ao coronavírus, com } \\
\text { diagnóstico ou tratamento de transtornos mentais até } 12 \text { meses antes da } \\
\text { pesquisa e com uma autoavaliação de saúde ruim. }\end{array}$ \\
\hline $\begin{array}{l}\text { Gutiérrez- } \\
\text { Hernández et al., } \\
2021\end{array}$ & $\begin{array}{l}\text { Estudo transversal. Analisar o estado de } \\
\text { saúde mental e o papel de variáveis } \\
\text { sociodemográficas e clínicas durante a } \\
\text { pandemia, além de averiguar se há uma } \\
\text { relação positiva entre autocompaixão e } \\
\text { um melhor estado de saúde mental. }\end{array}$ & $\begin{array}{l}\text { A pesquisa testou ansiedade, depressão e estresse em } 917 \text { participantes, } \\
\text { usando o Escala-21 de Depressão Ansiedade e Estresse (DASS-21), a } \\
\text { Escala de Autocompaixão (SCS) e o Questionário de Vulnerabilidade } \\
\text { Percebida à Doença (PVDQ) para avaliar o grau de risco percebido. } \\
\text { Cerca de } 30 \% \text { dos indivíduos pesquisados mostraram níveis clinicamente } \\
\text { significativos de ansiedade, depressão e estresse. As variáveis mais } \\
\text { frequentemente associadas a ansiedade, depressão e estresse foram } \\
\text { baixos níveis de autocompaixão, idade, sexo, sintomas físicos anteriores, } \\
\text { um transtorno mental anterior, ser estudante e vulnerabilidade percebida } \\
\text { à doença. }\end{array}$ \\
\hline $\begin{array}{l}\text { Limcaoco et al., } \\
2020\end{array}$ & $\begin{array}{l}\text { Pesquisa quantitativa. Avaliar estado } \\
\text { emocional atual da população em geral } \\
\text { por meio de uma pesquisa online. }\end{array}$ & $\begin{array}{l}\text { A pesquisa foi realizada com } 1.091 \text { respondentes de } 41 \text { países, de } 17 \text { de } \\
\text { março a } 1^{\circ} \text { de abril de } 2020 \text {. A média de idade dos respondentes foi de } \\
43,1 \text { anos com } 21,1 \% \text { sendo profissionais da saúde e mais de dois terços } \\
\text { eram mulheres. A Escala de Estresse Percebido (PSS-10) foi utilizada. } \\
\text { Escores significativamente mais altos foram observados entre mulheres, } \\
\text { jovens, estudantes e entre aqueles que expressaram preocupação em } \\
\text { ficarem infectados com COVID-19, além daqueles que perceberam } \\
\text { maior suscetibilidade ao coronavírus. Em contrapartida, não foram } \\
\text { observadas diferenças significativas entre os profissionais de saúde e a } \\
\text { população em geral. }\end{array}$ \\
\hline Liao et al., 2021 & $\begin{array}{l}\text { Estudo longitudinal prospectivo. } \\
\text { Avaliar o impacto da COVID-19 sobre } \\
\text { os sintomas depressivos subliminares e } \\
\text { identificar potenciais fatores atenuantes. }\end{array}$ & $\begin{array}{l}\text { Durante o surto de COVID-19, as condições e comportamentos de saúde } \\
\text { mental flutuaram de acordo com a progressão da pandemia. A maior taxa } \\
\text { de depressão e ansiedade prováveis foi encontrada após o pico de casos e } \\
\text { diminuiu com a remissão da doença. }\end{array}$ \\
\hline Liu et al., 2021 & $\begin{array}{l}\text { Estudo quantitativo. Estimar a } \\
\text { prevalência de depressão e ansiedade } \\
\text { em crianças e adolescentes após o } \\
\text { lockdown e identificar fatores } \\
\text { correlacionados durante o período de } \\
\text { lockdown da COVID-19. }\end{array}$ & $\begin{array}{l}\text { Dos participantes, } 12,33 \% \text { e } 6,26 \% \text { relataram depressão e ansiedade após } \\
\text { a quarentena, respectivamente. Ideação suicida, brigas com os pais, } \\
\text { insônia, dificuldade de concentração durante o aprendizado online e } \\
\text { humor ansioso e deprimido foram associados com depressão e ansiedade } \\
\text { após o isolamento. }\end{array}$ \\
\hline $\begin{array}{l}\text { Wang et al., } \\
2021\end{array}$ & $\begin{array}{l}\text { Pesquisa quantitativa. Avaliar estados } \\
\text { psicológicos públicos e seus fatores } \\
\text { relacionados durante a pandemia da } \\
\text { COVID-19. }\end{array}$ & $\begin{array}{l}\text { Foram recebidos } 600 \text { questionários válidos e utilizaou-se a Escala de } \\
\text { Autoavaliação de Ansiedade (SAS) e a Escala de Autoavaliação de } \\
\text { Depressão (SDS). O risco de ansiedade nas mulheres foi de } 3,01 \text { vezes } \\
\text { maior em comparação com os homens. Em comparação com as pessoas } \\
\text { com menos de } 40 \text { anos de idade, o risco de ansiedade de pessoas acima } \\
\text { de } 40 \text { anos de idade foi de } 0,40 \text { vezes maior. A taxa de ansiedade foi de } \\
6,33 \% \text { e depressão foi de } 17,17 \% \text {. }\end{array}$ \\
\hline
\end{tabular}

Fonte: Autores. 
Tabela 2 - Associação entre aspectos psicossociais e bruxismo ou DTM.

\begin{tabular}{|c|c|c|}
\hline Autor, ano & Método e objetivo & Resultados \\
\hline Alahmary, 2019 & $\begin{array}{l}\text { Estudo transversal. Investigar a prevalência de } \\
\text { DTM e sua associação com ansiedade e } \\
\text { depressão entre estudantes de odontologia em } \\
\text { Riade, Arábia Saudita. }\end{array}$ & $\begin{array}{l}\text { Dos estudantes avaliados, } 49,5 \% \text { apresentaram DTM. Destes, } \\
30,8 \% \text { apresentavam sinais de ansiedade e } 76,9 \% \text { apresentavam } \\
\text { sinais de depressão. Não houve associação entre DTM e idade, } \\
\text { mas houve uma maior prevalência no sexo feminino. }\end{array}$ \\
\hline $\begin{array}{l}\text { Almeida; } \\
\text { Guimarães; } \\
\text { Almeida, } 2018\end{array}$ & $\begin{array}{l}\text { Pesquisa bibliográfica exploratória. Abordar a } \\
\text { influência do estresse na manutenção de } \\
\text { agravos à saúde bucal, discorrendo sobre a } \\
\text { conduta do cirurgião-dentista diante desta } \\
\text { situação. }\end{array}$ & $\begin{array}{l}\text { A condição bucal associada ao estresse mais investigada foi a } \\
\text { DTM. A maioria dos estudos mostra que há uma associação entre } \\
\text { fatores psicoemocionais e DTM. }\end{array}$ \\
\hline $\begin{array}{l}\text { Canales et al., } \\
2019\end{array}$ & $\begin{array}{l}\text { Pesquisa quali-quantitativa. } \\
\text { Descrever a frequência de diagnósticos } \\
\text { psicossociais em uma grande amostra de } \\
\text { pacientes atendidos em uma clínica terciária de } \\
\text { DTM para fornecer uma base epidemiológica } \\
\text { para comparações futuras. }\end{array}$ & $\begin{array}{l}\text { A maioria dos pacientes apresentava uma deficiência baixa ou } \\
\text { nenhuma deficiência, com apenas uma pequena parte dos } \\
\text { indivíduos apresentando um comprometimento relacionado à dor } \\
\text { severamente limitante e de alta deficiência. Por outro lado, quase } \\
\text { metade dos indivíduos apresentaram níveis moderados a graves } \\
\text { de depressão e } 3 / 4 \text { apresentando níveis moderados a graves de } \\
\text { somatização. O sexo feminino apresentou o maior percentual de } \\
\text { valores anormais nas escalas de depressão e somatização. }\end{array}$ \\
\hline Yap; Natu, 2020 & $\begin{array}{l}\text { Estudo transversal observacional. Avaliar as } \\
\text { inter-relações entre DTM dolorosas, sintomas } \\
\text { somáticos e psicológicos em jovens asiáticos. }\end{array}$ & $\begin{array}{l}\text { O grupo com DTM apresentou sintomas somáticos moderados, } \\
\text { depressão moderada, ansiedade moderada a grave e estresse leve. } \\
\text { A prevalência de DTMs relacionadas à dor foi de } 8,5 \% \text {. } \\
\text { Indivíduos com DTM dolorosa apresentaram níveis } \\
\text { significativamente mais elevados de sofrimento somático e } \\
\text { psicológico. }\end{array}$ \\
\hline Zavanelli, 2017 & $\begin{array}{l}\text { Revisão de literatura. Apresentar revisão } \\
\text { sistematizada da literatura enfocando a } \\
\text { integração da DTM e psicologia. }\end{array}$ & $\begin{array}{l}\text { Os resultados obtidos evidenciaram a importância de uma } \\
\text { abordagem multiprofissional na assistência aos portadores de } \\
\text { DTM, a fim de proporcionar atendimento integral ao paciente } \\
\text { com essa disfunção. Apesar disso, a literatura disponível expondo } \\
\text { terapias psicológicas no tratamento da DTM é escassa, } \\
\text { demonstrando a carência de estudos nessa área. }\end{array}$ \\
\hline
\end{tabular}

Fonte: Autores. 
Tabela 3 - Associação entre as alterações psicológicas causadas pela pandemia por COVID-19 e sua relação com bruxismo e DTM.

\begin{tabular}{|c|c|c|}
\hline Autor, ano & Método e objetivo & Resultados \\
\hline $\begin{array}{l}\text { Asquini et al., } \\
2021\end{array}$ & $\begin{array}{l}\text { Estudo de coorte prospectivo. Compreender o } \\
\text { impacto da angústia relacionada a COVID-19 } \\
\text { nas variáveis gerais de saúde, comportamentos } \\
\text { orais, características psicossociais e } \\
\text { intensidade da dor facial em } 45 \text { pacientes } \\
\text { italianos com DTM. }\end{array}$ & $\begin{array}{l}\text { Os participantes com DTM crônica antes da pandemia de } \\
\text { COVID-19 apresentaram-se mais suscetíveis ao estresse } \\
\text { relacionado à COVID-19 em comparação com aqueles com DTM } \\
\text { aguda/subaguda. Em ambos os grupos, a ansiedade e a depressão } \\
\text { pioraram desde o início até o acompanhamento, sendo pior para } \\
\text { aqueles com DTM crônica. }\end{array}$ \\
\hline $\begin{array}{l}\text { Emodi-Perlman } \\
\text { et al., } 2020\end{array}$ & $\begin{array}{l}\text { Estudo transversal. Avaliar os efeitos da } \\
\text { pandemia por COVID-19 sobre os sintomas de } \\
\text { bruxismo e DTM entre os indivíduos de Israel } \\
\text { e Polônia e definir os preditores de bruxismo e } \\
\text { DTM durante o período de lockdown nesses } \\
\text { países. }\end{array}$ & $\begin{array}{l}\text { A pandemia por COVID-19 causou efeitos adversos significativos } \\
\text { no estado psicoemocional em ambas populações (israelenses e } \\
\text { poloneses), resultando na intensificação do seu bruxismo e } \\
\text { sintomas de DTM, levando ao aumento da dor orofacial. }\end{array}$ \\
\hline $\begin{array}{l}\text { Gaş; } \quad \text { Özsoy; } \\
\text { Aydin, } 2021\end{array}$ & $\begin{array}{l}\text { Estudo transversal. Caracterizar o efeito da } \\
\text { pandemia por COVID-19 na mudança de } \\
\text { níveis de qualidade do sono, estresse e } \\
\text { ansiedade e sua relação com disfunção } \\
\text { temporomandibular em estudantes de } \\
\text { odontologia. }\end{array}$ & $\begin{array}{l}\text { A incidência de disfunção temporomandibular foi de } 77,5 \% \text {. } \\
\text { Ansiedade, depressão e os escores de estresse de estudantes do } \\
\text { sexo feminino foram significativamente maiores do que os do } \\
\text { sexo masculino. Apesar do estudo observar um aumento na } \\
\text { qualidade do sono, a pandemia de COVID-19 provocou maior } \\
\text { número de casos de DTM, depressão, ansiedade e níveis de } \\
\text { estresse entre na população estudada. }\end{array}$ \\
\hline $\begin{array}{l}\text { Medeiros et al., } \\
2020\end{array}$ & $\begin{array}{l}\text { Pesquisa quantitativa. Avaliar a prevalência de } \\
\text { sintomas de DTM, ansiedade, depressão, } \\
\text { comportamentos orais e suas associações } \\
\text { durante o isolamento social devido à pandemia } \\
\text { por COVID-19. }\end{array}$ & $\begin{array}{l}\text { Observou-se alta prevalência de sintomas de DTM e alto índice } \\
\text { de sintomas de ansiedade }(49,6 \%) \text { e depressão }(38,9 \%) \text {. Houve } \\
\text { correlação positiva entre comportamentos orais e sintomas de } \\
\text { DTM, ansiedade e depressão. }\end{array}$ \\
\hline $\begin{array}{l}\text { Restrepo et al., } \\
2021\end{array}$ & $\begin{array}{l}\text { Estudo transversal. Avaliar a associação entre } \\
\text { fatores psicológicos (sintomas de ansiedade, } \\
\text { depressão e somatização) e DTM em } \\
\text { adolescentes entre } 12 \text { e } 15 \text { anos, pertencentes } \\
\text { às zonas urbana e rural da Colômbia. }\end{array}$ & $\begin{array}{l}\text { Cerca de } 40 \% \text { das pessoas incluídas no estudo apresentavam } \\
\text { algum tipo de DTM. As DTMs relacionadas à dor foram as mais } \\
\text { encontradas, sendo a mialgia a mais comum. Não houve } \\
\text { disparidade em relação ao sexo, mas houve diferença nos } \\
\text { sintomas de ansiedade e somatização. A DTM foi mais prevalente } \\
\text { em crianças com } 13 \text { anos de idade, enquanto os fatores } \\
\text { psicológicos estiveram presentes em todas as faixas etárias. Uma } \\
\text { associação estatisticamente significativa entre DTM e sintomas de } \\
\text { ansiedade, depressão e somatização foi encontrada em indivíduos } \\
\text { da zona rural. }\end{array}$ \\
\hline $\begin{array}{l}\text { Saccomanno et } \\
\text { al., } 2020\end{array}$ & $\begin{array}{l}\text { Pesquisa quantitativa. Investigar a presença de } \\
\text { sintomas relacionados às DTM e o tempo de } \\
\text { aparecimento e agravamento dos sintomas } \\
\text { dolorosos em relação às mudanças na vida } \\
\text { social impostas pela pandemia do coronavírus } \\
\text { e avaliar a percepção do COVID-19 como um } \\
\text { grande evento estressante em indivíduos que } \\
\text { relatam piora dos sintomas dolorosos de DTM. }\end{array}$ & $\begin{array}{l}\text { Dentre os indivíduos estudados, } 40,7 \% \text { reclamaram de sintomas } \\
\text { de DTM no mês anterior à pesquisa. Desses, } 74,3 \% \text { dos } \\
\text { indivíduos que reclamaram de dores na face, mandíbula ou } \\
\text { têmpora no último mês eram mulheres. } \\
\text { No que diz respeito ao tempo de início, } 60,8 \% \text { deles relataram } \\
\text { que a dor facial teve início nos últimos três meses, enquanto } \\
51,4 \% \text { desses indivíduos relataram que seus sintomas pioraram no } \\
\text { último mês e afirmaram estar relacionado ao estresse vivenciado } \\
\text { durante a quarentena. }\end{array}$ \\
\hline Silva et al. 2021 & $\begin{array}{l}\text { Revisão de literatura. Realizar uma revisão de } \\
\text { literatura acerca da relação dos sintomas de } \\
\text { bruxismo e disfunção temporomandibular e a } \\
\text { ansiedade ocasionada pela pandemia de } \\
\text { COVID-19. }\end{array}$ & $\begin{array}{l}\text { A pandemia da COVID-19 e o isolamento social pode estar } \\
\text { associada ao aumento de sintomas psicológicos como ansiedade e } \\
\text { depressão. Esses sintomas possuem associação com as disfunções } \\
\text { temporomandibulares e bruxismo levando ao surgimento e } \\
\text { desenvolvimento de ambas afecções. }\end{array}$ \\
\hline
\end{tabular}




\section{Discussão}

Dos artigos incluídos nesta revisão, 7 abordavam aspectos psicossociais e sua relação com a pandemia por COVID-19 (Di Blasi et al., 2021; De Boni et al., 2020; Gutiérrez-Hernández et al., 2021; Limcaoco et al., 2020; Liao et al., 2021; Liu et al., 2021; Wang et al., 2021), 5 apresentavam os aspectos psicossociais e sua relação com DTM (Alahmary, 2019; Almeida; Guimarães; Almeida, 2018; Canales et al., 2019; Yap; Natu, 2020; Zavanelli, 2017) e 7 dissertavam sobre a associação entre as alterações psicológicas causadas pela pandemia por COVID-19 e sua relação com bruxismo e DTM (Asquini et al., 2021; Emodi-Perlman et al., 2020; Gaş; Özsoy; Aydin, 2021; Medeiros et al., 2020; Restrepo et al., 2021; Saccomanno et al., 2020; Silva et al. 2021).

\section{Aspectos psicossociais e a pandemia por COVID-19}

Mudanças críticas no cotidiano de comunidades tendem a gerar distúrbios, sejam eles sociais ou psicológicos. A pandemia por COVID-19 surgiu como um problema de nível global afetando a todos abruptamente. A perspectiva e a necessidade do isolamento social acabaram por provocar um sentimento de impotência, solidão e de forte angústia individual (Di Blasi et al., 2021; Limcaoco et al., 2020; Pereira et al., 2020).

Liao et al. (2021) observaram que as condições de saúde mental e comportamentos oscilaram de acordo com a evolução da pandemia. As maiores taxas de depressão e ansiedade foram encontradas após o pico de casos e regrediram com a diminuição dos casos da doença. Em seu estudo, Liu et al. (2021) encontraram que 12,33\% e 6,26\% relataram depressão e ansiedade após a quarentena, respectivamente. Foram relatadas várias condições durante o período de isolamento, como: insônia, humor ansioso e deprimido, dificuldade de atenção durante o estudo ou trabalho, briga com parentes e até ideações suicidas, que vão de acordo com os dados encontrados na pesquisa de Pereira et al. (2020).

Gutíerrez-Hernández et al. (2021) realizaram uma pesquisa com 917 pessoas, onde encontraram que 30\% destas apresentaram níveis clinicamente significativos de ansiedade, depressão e estresse. Wang et al. (2021) afirmaram que o risco de ansiedade nas mulheres é de 3,01 vezes maior quando comparado ao sexo masculino. Em concordância com os resultados de Wang et al. (2021), De Boni et al. (2020) observaram que os indivíduos mais propensos a desenvolverem sintomas de ansiedade ou depressão foram mulheres jovens trabalhadoras da linha de frente de combate ao COVID-19.

Assim, a partir dos resultados encontrados nos estudos, observou-se relação direta entre o isolamento social e o surgimento ou agravamento de sintomas de depressão, ansiedade e estresse na população.

\section{Aspectos psicossociais e a sua relação com DTM}

O estresse está intimamente relacionado à condição bucal, principalmente à disfunção temporomandibular. É comprovado que há uma relação entre fatores psicoemocionais e DTM (Almeida; Guimarães; Almeida, 2018). Alahmary (2019) investigou a prevalência de DTM e sua relação com ansiedade e depressão em estudantes de odontologia da Arábia Saudita e constatou que 49,5\% tinham DTM e destes, 30,8\% e 76,9\% manifestaram sinais de ansiedade e depressão, respectivamente, havendo maior prevalência no sexo feminino. Canales et al. (2019) também encontraram maior percentual de depressão e somatização em mulheres.

Portadores de DTM com sintomas dolorosos apresentaram níveis significativamente maiores de sofrimento somático e psicológico (Yap; Natu, 2020). Assim, Zavanelli et al. (2017) ressaltaram a importância de uma abordagem multiprofissional no cuidado desses indivíduos a fim de garantir um atendimento integralizado. 


\section{Associação entre as alterações psicológicas causadas pela pandemia por COVID-19 e sua relação com o bruxismo e DTM}

A pandemia por COVID-19 e o consequente isolamento social pode estar relacionado ao maior número de sintomas psicológicos, como a ansiedade e a depressão. Sabe-se que esses sintomas possuem associação direta com bruxismo e DTM, levando ao surgimento e progressão dessas alterações (Silva et al., 2021). Emodi-Perlman et al. (2020) afirmaram que a pandemia provocou efeitos adversos significativos no estado psicoemocional das populações estudadas, relatando intensificação do bruxismo dos sintomas de DTM além do aumento da dor orofacial.

Medeiros et al. (2020) constataram alta prevalência de sintomas de DTM e índices elevados de ansiedade (49,6\%) e depressão $(38,9 \%)$. Além disso, houve correspondência positiva entre comportamentos orais e sintomas de DTM, ansiedade e depressão. Com a pandemia do coronavírus houve um aumento de casos de DTM. Gaş, Özsoy e Aydin (2021) caracterizaram o efeito da pandemia em estudantes de odontologia e verificaram que 77,5\% desses apresentaram DTM. Além disso, observaram que apesar de ter ocorrido um aumento da qualidade do sono, houve maior número de casos de depressão, ansiedade e níveis de estresse da população estudada. Na amostra do estudo, ansiedade, depressão e estresse foram estatisticamente mais significativas em estudantes do sexo feminino. Em concordância, Saccomanno et al. (2020) relataram que 40,7\% dos indivíduos da pesquisa reclamaram de sintomas de DTM no mês anterior à realização da pesquisa, desses, $74,3 \%$ eram mulheres.

Já Restrepo et al. (2021) avaliaram que dos sujeitos incluídos no seu estudo, 40\% apresentaram algum tipo de DTM. As mais encontradas foram as DTMs relacionadas à dor, sendo a mialgia a mais comum. Não houve diferença significativa entre homens e mulheres em relação a sintomatologia de DTM.

Para entender o impacto do COVID-19 na saúde bucal da população, Asquini et al. (2021) realizaram uma pesquisa com 45 italianos com DTM. Dentre estes, os que possuíam a DTM crônica eram mais frágeis e suscetíveis ao estresse da pandemia em comparação com os que possuíam DTM aguda/subaguda. Porém, em ambos os grupos, a ansiedade e depressão aumentaram desde o início até o final da pesquisa, entretanto, os valores foram piores para os pacientes de DTM crônica.

\section{Considerações Finais}

A pandemia por COVID-19 provocou muitas adversidades, resultando em prejuízos à saúde mental. O isolamento social, o medo pela doença e a mudança na rotina causou elevação dos níveis de estresse, ansiedade e depressão na população em geral. Observou-se que tais alterações têm correlação direta com agravos de sintomas do bruxismo e da DTM, resultando no aumento da incidência dessas desordens bucais e no agravamento de condições preexistentes.

\section{Referências}

Alahmary, A. W. (2019). Association of temporomandibular disorder symptoms with anxiety and depression in Saudi dental students. Open access Macedonian journal of medical sciences, 7(23), 4116.

Almeida, R. S., de Lima Guimarães, J., \& de Almeida, J. Z. (2018). Estresse emocional e sua influência na saúde bucal. DêCiência em Foco, 2 (1), 78-102.

Almeida-Leite, C. M., Stuginski-Barbosa, J., \& Conti, P. C. R. (2020). How psychosocial and economic impacts of COVID-19 pandemic can interfere on bruxism and temporomandibular disorders? Journal of Applied Oral Science, 28.

Aquino, E. M., Silveira, I. H., Pescarini, J. M., Aquino, R., \& Souza-Filho, J. A. D. (2020). Medidas de distanciamento social no controle da pandemia de COVID-19: potenciais impactos e desafios no Brasil. Ciência \& Saúde Coletiva, 25, 2423-2446.

Asquini, G., Bianchi, A. E., Borromeo, G., Locatelli, M., \& Falla, D. (2021). The impact of Covid-19-related distress on general health, oral behaviour, psychosocial features, disability and pain intensity in a cohort of Italian patients with temporomandibular disorders. Plos one, 16(2), e0245999.

Ather, A., Patel, B., Ruparel, N. B., Diogenes, A., \& Hargreaves, K. M. (2020). Coronavirus disease 19 (COVID-19): implications for clinical dental care. Journal of endodontics, 46(5), 584-595. 
Boni, R. B., Balanzá-Martínez, V., Mota, J. C., Cardoso, T. D. A., Ballester, P., Atienza-Carbonell, B., ... \& Kapczinski, F. (2020). Depression, Anxiety, and Lifestyle Among Essential Workers: A Web Survey from Brazil and Spain During the COVID-19 Pandemic. Journal of medical Internet research, 22(10), e22835.

Canales, G. D. L. T., Guarda-Nardini, L., Rizzatti-Barbosa, C. M., Conti, P. C. R., \& Manfredini, D. (2019). Distribution of depression, somatization and painrelated impairment in patients with chronic temporomandibular disorders. Journal of Applied Oral Science, 27.

Di Blasi, M., Gullo, S., Mancinelli, E., Freda, M. F., Esposito, G., Gelo, O. C. G., ... \& Coco, G. L. (2021). Psychological distress associated with the COVID19 lockdown: A two-wave network analysis. Journal of affective disorders, 284, 18-26.

Emodi-Perlman, A., Eli, I., Smardz, J., Uziel, N., Wieckiewicz, G., Gilon, E., ... \& Wieckiewicz, M. (2020). Temporomandibular Disorders and Bruxism Outbreak as a Possible Factor of Orofacial Pain Worsening during the COVID-19 Pandemic - Concomitant Research in Two Countries. Journal of clinical medicine, 9(10), 3250 .

Gaş, S., Ekşi Özsoy, H., \& Cesur Aydın, K. (2021). The association between sleep quality, depression, anxiety and stress levels, and temporomandibular joint disorders among Turkish dental students during the COVID-19 pandemic. CRANIO, 1-6.

Gutiérrez-Hernández, M. E., Fanjul, L. F., Díaz-Megolla, A., Reyes-Hurtado, P., Herrera-Rodríguez, J. F., Enjuto-Castellanos, M. D. P., \& Peñate, W. (2021). Covid-19 lockdown and mental health in a sample population in spain: the role of self-compassion. International journal of environmental research and public health, 18(4), 2103 .

Izzetti, R., Nisi, M., Gabriele, M., \& Graziani, F. (2020). COVID-19 transmission in dental practice: brief review of preventive measures in Italy. Journal of dental research, 99(9), 1030-1038.

Liao, Y. H., Fan, B. F., Zhang, H. M., Guo, L., Lee, Y., Wang, W. X., ... \& McIntyre, R. S. (2021). The impact of COVID-19 on subthreshold depressive symptoms: a longitudinal study. Epidemiology and psychiatric sciences, 30.

Limcaoco, R. S. G., Mateos, E. M., Fernandez, J. M., \& Roncero, C. (2020). Anxiety, worry and perceived stress in the world due to the COVID-19 pandemic, March 2020. Preliminary results. MedRxiv.

Liu, Y., Yue, S., Hu, X., Zhu, J., Wu, Z., Wang, J., \& Wu, Y. (2021). Associations between feelings/behaviors during COVID-19 pandemic lockdown and depression/anxiety after lockdown in a sample of Chinese children and adolescents. Journal of Affective Disorders, 284, 98-103.

Medeiros, R. A. D., Vieira, D. L., Silva, E. V. F. D., Rezende, L. V. M. D. L., Santos, R. W. D., \& Tabata, L. F. (2020). Prevalence of symptoms of temporomandibular disorders, oral behaviors, anxiety, and depression in Dentistry students during the period of social isolation due to COVID-19. Journal of Applied Oral Science, 28.

OPAS. (2020) Folha informativa COVID-19 - Escritório da OPAS e da OMS no Brasil. Recuperado em 07/04/2021, de https://www.paho.org/pt/covid19.

Pereira, M. D., de Oliveira, L. C., Costa, C. F. T., de Oliveira Bezerra, C. M., Pereira, M. D., dos Santos, C. K. A., \& Dantas, E. H. M. (2020). A pandemia de COVID-19, o isolamento social, consequências na saúde mental e estratégias de enfrentamento: uma revisão integrativa. Research, Society and Development, 9(7), e652974548-e652974548.

Restrepo, C., Ortiz, A. M., Henao, A. C., \& Manrique, R. (2021). Association between psychological factors and temporomandibular disorders in adolescents of rural and urban zones. BMC Oral Health, 21(1), 1-11.

Saccomanno, S., Bernabei, M., Scoppa, F., Pirino, A., Mastrapasqua, R., \& Visco, M. A. (2020). Coronavirus Lockdown as a Major Life Stressor: Does It Affect TMD Symptoms? International Journal of Environmental Research and Public Health, 17(23), 8907.

Silva, E. et al (2021). A relação dos sintomas de bruxismo e disfunção temporomandibular e a ansiedade ocasionada pela pandemia da COVID-19: uma revisão de literatura. Research, Society and Development, 10(2).

Tassara, I. G., Okabayashi, N. Y. T., Casaca, M. C. G., \& de Souza Veronez, F. (2021). Prevalência de sintomas psicológicos em tempos de isolamento social. Brazilian Journal of Health Review, 4(1), 1295-1309.

Wang, Y., Di, Y., Ye, J., \& Wei, W. (2021). Study on the public psychological states and its related factors during the outbreak of coronavirus disease 2019 (COVID-19) in some regions of China. Psychology, health \& medicine, 26(1), 13-22.

Yap, A. U., \& Natu, V. P. (2020). Inter-relationships between pain-related temporomandibular disorders, somatic and psychological symptoms in Asian youths. Journal of Oral Rehabilitation, 47(9), 1077-1083.

Zavanelli, A. C., Rezende, M. C. R. A., dos Santos-Neto, O. M., \& Fajardo, R. S. (2017). Integração da Psicologia e Odontologia na DTM: revisão sistematizada. Archives of health investigation, 6(11). 